MATHEMATICS OF COMPUTATION

Volume 76, Number 260, October 2007, Pages 2181-2187

S $0025-5718(07) 01972-2$

Article electronically published on April 17, 2007

\title{
STRUCTURE OF GRÖBNER BASES WITH RESPECT TO BLOCK ORDERS
}

\author{
M'HAMMED EL KAHOUI AND SAID RAKRAK
}

\begin{abstract}
In this paper we study the structure of Gröbner bases with respect to block orders. We extend Lazard's theorem and the Gianni-Kalkbrenner theorem to the case of a zero-dimensional ideal whose trace in the ring generated by the first block of variables is radical. We then show that they do not hold for general zero-dimensional ideals.
\end{abstract}

\section{INTRODUCTION}

The concept of Gröbner bases, introduced by Buchberger [6] in 1965, is nowadays one of the main tools for studying algebraic systems and various related problems in computational algebra; see [7] and the standard reference books [5, 2, 8, 15] for basic facts and applications of such a concept.

An important question is to understand the structure of Gröbner bases. In the case of two variables, Lazard [13] gave a complete structural understanding of lexicographic Gröbner bases. This result is extended to the case of univariate polynomial rings over Dedekind domains in 3. In the case of more than two variables an extension of Lazard's theorem is given by Marinari and Mora [14] in the case of radical zero-dimensional ideals.

Another important result is proved by Gianni [10] and Kalkbrenner [12 for lexicographic Gröbner bases of zero-dimensional ideals. More precisely, the result states that if $G$ is a Gröbner basis of a zero-dimensional ideal $\mathcal{I}$ in the polynomial ring $\mathbb{K}[x]=\mathbb{K}\left[x_{1}, \ldots, x_{n}\right]$ and if $\left(\mu_{1}, \ldots, \mu_{n-1}\right)$ is a zero of $\mathcal{I} \cap \mathbb{K}\left[x_{1}, \ldots, x_{n-1}\right]$, then the list of polynomials obtained from $G$ after substituting the $\mu_{i}$ 's to the $x_{i}$ 's already contains a gcd of the nonzero polynomials in it. This result is extended by Becker in [4, for the radical zero-dimensional case, in the sense that for any $i \leq n-1$ and any zero $\left(\mu_{1}, \ldots, \mu_{i}\right)$ of $\mathcal{I} \cap \mathbb{K}\left[x_{1}, \ldots, x_{i}\right]$ the image of $\mathcal{I}$ under the specialization homomorphism $\phi: x_{i} \longmapsto \mu_{i}$ has $\phi(G)$ as a Gröbner basis.

We are concerned in this paper with the question of understanding the structure of Gröbner bases with respect to block orders (see section 2 for the definition of block orders). We give extensions of Lazard's theorem and Becker's extension of the Gianni-Kalkbrenner theorem. More precisely, let $J=x_{1}, \ldots, x_{j}$ be a sub-list of $x$ and $\prec$ be a monomial block order built out of monomial orders on $J$ and its complement $J^{c}$. Given a zero-dimensional ideal $\mathcal{I}$ of $\mathbb{K}[x]$ such that $\mathcal{I} \cap \mathbb{K}[J]$ is radical, we show that $\mathcal{I}$ has a Gröbner basis which exhibits a factorized form

Received by the editor October 28, 2004 and, in revised form, March 22, 2006.

2000 Mathematics Subject Classification. Primary 13P10, 12 Y05.

Key words and phrases. Gröbner basis, Lazard structure theorem, Gianni-Kalkbrenner structure theorem.

(C)2007 American Mathematical Society 2181 
similar to the one in Lazard's theorem. We also show that Becker's extension of the Gianni-Kalkbrenner theorem holds in this case. We finally show that these results no longer hold in general if $\mathcal{I} \cap \mathbb{K}[J]$ is not radical (even for lexicographic orders).

\section{Notation AND BASiC FACTS}

Throughout this paper we will denote by $\mathbb{K}\left[x_{1}, \ldots, x_{n}\right]=\mathbb{K}[x]$ the ring of polynomials in $n$ indeterminates with coefficients in a commutative field $\mathbb{K}$. The multiplicative semigroup generated by $x=x_{1}, \ldots, x_{n}$ is denoted by $\mathbb{M}$, and its divisibility partial order by $\mid$.

For any sub-list $J$ of $x$, with $|J|$ as the number of elements and $J^{c}$ as the complement, we let $\mathbb{M}[J]$ be the sub-semigroup of $\mathbb{M}$ generated by $J$. Identified to $\mathbb{K}[J]\left[J^{c}\right]$, the ring $\mathbb{K}[x]$ has a natural structure of the free $\mathbb{K}[J]$-module with $\mathbb{M}\left[J^{c}\right]$ as the canonical basis. Thus, any polynomial $f \in \mathbb{K}[x]$ is uniquely written as

$$
f=\sum a_{\alpha} x^{\alpha}
$$

where the sum ranges over a finite subset of $\mathbb{M}\left[J^{c}\right]$ and the $a_{\alpha}$ 's are elements of $\mathbb{K}[J]$.

Given an admissible order $\prec$ on the monomials of $\mathbb{M}$ we define the leading term of $f$ with respect to $J$ and $\prec$, denoted by $\operatorname{Lt}(f, J, \prec)$, to be $a_{\beta} x^{\beta}$ where $x^{\beta}$ is the greatest element of $\left\{x^{\alpha} \in \mathbb{M}\left[J^{c}\right] ; a_{\alpha} \neq 0\right\}$ with respect to $\prec$. The monomial $x^{\beta}$ is called the leading monomial of $f$ with respect to $J$ and $\prec$ and is denoted by $\operatorname{Lm}(f, J, \prec)$. The coefficient $a_{\beta}$ is called the leading coefficient of $f$ with respect to $J$ and $\prec$ and is denoted by $\operatorname{Lc}(f, J, \prec)$.

Let $\mathcal{I}$ be an ideal of $\mathbb{K}[x], J$ be a sub-list of $x$ and $\prec$ be an admissible order on the monomials. Given $x^{\alpha} \in \mathbb{M}\left[J^{c}\right]$ we let $\mathcal{J}\left(\mathcal{I}, J, \prec, x^{\alpha}\right)$ be the set

$$
\left\{a \in \mathbb{K}[J] ; a=0 \text { or }\left(\exists f \in \mathcal{I} \quad \operatorname{Lm}(f, J, \prec)=x^{\alpha}, \operatorname{Lc}(f, J, \prec)=a\right)\right\} .
$$

This is obviously an ideal of $\mathbb{K}[J]$ and the sequence $\left(\mathcal{J}\left(\mathcal{I}, J, \prec, x^{\alpha}\right)\right)_{\alpha}$ is non-decreasing, i.e. $\mathcal{J}\left(\mathcal{I}, J, \prec, x^{\alpha}\right) \subseteq \mathcal{J}\left(\mathcal{I}, J, \prec, x^{\beta}\right)$ whenever $x^{\alpha} \mid x^{\beta}$.

Given any sub-list $J$ of $x$ and a finite subset $G$ of $\mathbb{K}[x]$ we will write $G=$ $\left\{B_{1, i}, \ldots, B_{r_{i}, i} ; i=0, \ldots, s\right\}$, where $\operatorname{Lm}\left(B_{j, i}, J, \prec\right)=x^{\alpha^{(i)}}$ and $x^{\alpha^{(0)}} \prec x^{\alpha^{(1)}} \prec$ $\cdots \prec x^{\alpha^{(s)}}$.

The following definition of block orders and their main properties can be found in $[8$.

Definition 2.1. Given an admissible order $\prec$ on $\mathbb{M}$ and a sub-list $J$ of $x$, we will say that $\prec$ is a block order with respect to $J$ if for any $a, a^{\prime} \in \mathbb{M}[J]$ and $b, b^{\prime} \in \mathbb{M}\left[J^{c}\right]$ we have:

$$
a b \prec a^{\prime} b^{\prime} \Leftrightarrow b \prec b^{\prime} \text { or }\left(b=b^{\prime} \text { and } a \prec a^{\prime}\right) .
$$

A typical example of block order is given by the lexicographic order $x_{1} \prec_{\text {lex }}$ $x_{2} \prec_{\text {lex }} \cdots \prec_{\text {lex }} x_{n}$ and $J=x_{1}, \ldots, x_{i}$, where $i \leq n-1$. In general, let $J$ be a sub-list of $x$ and $\prec_{J}\left(\right.$ resp. $\prec_{J^{c}}$ ) be an admissible order on $\mathbb{M}[J]$ (resp. $\mathbb{M}\left[J^{c}\right]$ ). If $\prec$ is the lexicographic order on $\mathbb{M}=\mathbb{M}[J] \times \mathbb{M}\left[J^{c}\right]$ built with $\left(\mathbb{M}[J], \prec_{J}\right)$ and $\left(\mathbb{M}\left[J^{c}\right], \prec J^{c}\right)$, then $\prec$ is a block order with respect to $J$.

If $\prec$ is a block order with respect to $J$, then for any polynomial $f \in \mathbb{K}[x]$ we have:

$$
\operatorname{Lm}(f, \prec)=\operatorname{Lm}(\operatorname{Lc}(f, J, \prec), \prec) \operatorname{Lm}(f, J, \prec)
$$


We will also need the following classical result; see [1] for a more general result.

Theorem 2.2. Let $\mathcal{I}$ be an ideal of $\mathbb{K}[x]$ and $J$ be a sub-list of $x$. Let $\prec$ be a block order with respect to $J$. Then, for any finite subset $G$ of $\mathcal{I}$ the following assertions are equivalent:

i) $G$ is a Gröbner basis of the ideal $\mathcal{I}$ with respect to $\prec$,

ii) for any $x^{\alpha} \in \mathbb{M}\left[J^{c}\right]$ the set

$$
\left\{\operatorname{Lc}(f, J, \prec) ; f \in G, \operatorname{Lm}(f, J, \prec) \mid x^{\alpha}\right\}
$$

is a Gröbner basis of the ideal $\mathcal{J}\left(\mathcal{I}, J, \prec, x^{\alpha}\right)$ with respect to $\prec J$.

Proof. "i) $\Rightarrow$ ii)" Let $c \in \mathcal{J}\left(\mathcal{I}, J, \prec, x^{\alpha}\right)$ and $f \in \mathcal{I}$ be $\operatorname{such}$ that $\operatorname{Lm}(f, J, \prec)=x^{\alpha}$ and $\operatorname{Lc}(f, J, \prec)=c$. Since $G$ is a Gröbner basis of $\mathcal{I}$ with respect to $\prec$ there exists $g \in G$ such that $\operatorname{Lm}(g, \prec) \mid \operatorname{Lm}(f, \prec)$.

According to the relation (2.1) we have

$$
\begin{aligned}
& \operatorname{Lm}(g, J, \prec) \mid \operatorname{Lm}(f, J, \prec)=x^{\alpha}, \\
& \operatorname{Lm}(\operatorname{Lc}(g, J, \prec), \prec) \mid \operatorname{Lm}(\operatorname{Lc}(f, J, \prec), \prec)=\operatorname{Lm}(c, \prec J) .
\end{aligned}
$$

Therefore, the set $\left\{\operatorname{Lc}(g, J, \prec) ; g \in G\right.$ and $\left.\operatorname{Lm}(g, J, \prec) \mid x^{\alpha}\right\}$ is a Gröbner basis of the ideal $\mathcal{J}\left(\mathcal{I}, J, \prec, x^{\alpha}\right)$ with respect to $\prec_{J}$.

"ii) $\Rightarrow$ i)" Let $f \in \mathcal{I}$ and write $\operatorname{Lm}(f, J, \prec)=x^{\alpha}$. Since the set $\{\operatorname{Lc}(g, J, \prec) ; g \in$ $G$ and $\left.\operatorname{Lm}(g, J, \prec) \mid x^{\alpha}\right\}$ is a Gröbner basis of the ideal $\mathcal{J}\left(\mathcal{I}, J, \prec, x^{\alpha}\right)$ with respect to $\prec_{J}$ there exists $g \in G$ such that

$$
\begin{aligned}
& \operatorname{Lm}(g, J, \prec) \mid \operatorname{Lm}(f, J, \prec)=x^{\alpha}, \\
& \operatorname{Lm}(\operatorname{Lc}(g, J, \prec), \prec) \mid \operatorname{Lm}(\operatorname{Lc}(f, J, \prec), \prec) .
\end{aligned}
$$

Therefore, $\operatorname{Lm}(g, \prec) \mid \operatorname{Lm}(f, \prec)$ according to the relation (2.1). This shows that $G$ is a Gröbner basis of $\mathcal{I}$ with respect to $\prec$.

\section{THE CASE OF ZERO-DIMENSIONAL IDEALS WITH RADICAL TRACE ON THE FIRST BLOCK}

Let $J$ be a sub-list of $x$ and $\prec$ be a block order with respect to $J$. In this section we study the structure of a zero-dimensional ideal $\mathcal{I}$ of $\mathbb{K}[x]$ as a $\mathbb{K}[J]$-submodule of $\mathbb{K}[J]\left[J^{c}\right]$. Under the additional assumption that $\mathcal{I} \cap \mathbb{K}[J]$ is radical, we give an extension of Lazard's theorem and we show that Becker's extension of the Gianni-Kalkbrenner theorem also holds in this case.

Lemma 3.1. Let $\mathcal{I}$ be a zero-dimensional ideal of $\mathbb{K}[x]$, $J$ be a sub-list of $x$ and $\prec$ be a block order with respect to $J$. Assume that the ideal $\mathcal{I} \cap \mathbb{K}[J]$ is radical. Then for any $x^{\alpha} \in \mathbb{M}\left[J^{c}\right]$ there exists a polynomial $H_{\alpha} \in \mathbb{K}[x]$ such that:

i) $\operatorname{Lm}\left(H_{\alpha}, J, \prec\right)=x^{\alpha}$ and $\operatorname{Lc}\left(H_{\alpha}, J, \prec\right)=1$,

ii) for any $c \in \mathcal{J}\left(\mathcal{I}, J, \prec, x^{\alpha}\right)$ we have $c H_{\alpha} \in \mathcal{I}$,

iii) for any $f \in \mathcal{I}$ there exists a unique sequence $\left(c_{\alpha}\right)_{x^{\alpha} \in \mathbb{M}\left[J^{c}\right]}$, having a finite support, such that $f=\sum c_{\alpha} H_{\alpha}$ and $c_{\alpha} \in \mathcal{J}\left(\mathcal{I}, J, \prec, x^{\alpha}\right)$.

Proof. To simplify let $\mathcal{J}_{\alpha}=\mathcal{J}\left(\mathcal{I}, J, \prec, x^{\alpha}\right)$, and notice that $\mathcal{J}_{0}=\mathcal{I} \cap \mathbb{K}[J]$ is radical zero-dimensional and $\mathcal{J}_{0} \subset \mathcal{J}_{\alpha}, \mathcal{J}_{0} \subset \mathcal{J}_{0}: \mathcal{J}_{\alpha}$ for any $\alpha$. This implies in particular that $\mathcal{J}_{\alpha}$, as well as $\mathcal{J}_{0}: \mathcal{J}_{\alpha}$, is either radical zero-dimensional or equal to $\mathbb{K}[J]$. Moreover we have $\left(\mathcal{J}_{0}: \mathcal{J}_{\alpha}\right)+\mathcal{J}_{\alpha}=\mathbb{K}[J]$.

Let $a \in \mathcal{J}_{0}: \mathcal{J}_{\alpha}$ and $b \in \mathcal{J}_{\alpha}$ be such that $a+b=1$, and let $f \in \mathcal{I}$ be such that $\operatorname{Lm}(f, J, \prec)=x^{\alpha}$ and $\operatorname{Lc}(f, J, \prec)=b$. If we let $H_{\alpha}=f+a x^{\alpha}$, then we have 
$\operatorname{Lm}\left(H_{\alpha}, J, \prec\right)=x^{\alpha}$ and $\operatorname{Lc}\left(H_{\alpha}, J, \prec\right)=a+b=1$. On the other hand, given any $c \in \mathcal{J}_{\alpha}$ we have $c a \in \mathcal{J}_{0} \subset \mathcal{I}$ and hence $c H_{\alpha}=c f+c a x^{\alpha}$ belongs to the ideal $\mathcal{I}$.

Let $f \in \mathcal{I} \backslash\{0\}, x^{\alpha^{(0)}}=\operatorname{Lm}(f, J, \prec)$ and $c_{\alpha^{(0)}}=\operatorname{Lc}(f, J, \prec)$. Then $f_{1}=f-$ $c_{\alpha^{(0)}} H_{\alpha^{(0)}}$ belongs to $\mathcal{I}$ and satisfies $x^{\alpha^{(1)}}=\operatorname{Lm}\left(f_{1}, J, \prec\right) \prec \operatorname{Lm}(f, J, \prec)$. If $f_{1} \neq 0$, then we repeat the process to construct another polynomial

$$
f_{2}=f_{1}-\operatorname{Lc}\left(f_{1}, J, \prec\right) H_{\alpha^{(1)}}
$$

which satisfies the relation $x^{\alpha^{(2)}}=\operatorname{Lm}\left(f_{2}, J, \prec\right) \prec \operatorname{Lm}\left(f_{1}, J, \prec\right)$. Continuing this way we construct a sequence $f_{0}=f, f_{1}, \ldots, f_{t}, \ldots$ in $\mathcal{I}$ such that

$$
\operatorname{Lm}\left(f_{0}, J, \prec\right) \succ \operatorname{Lm}\left(f_{1}, J, \prec\right) \succ \cdots \operatorname{Lm}\left(f_{t}, J, \prec\right) \succ \cdots .
$$

According to the Artinian nature of the order $\prec$, the constructed sequence $\left(f_{i}\right)_{i \geq 0}$ should stop at some $t$. If $f_{t} \neq 0$, then we can construct another polynomial $f_{t+1}=$ $f_{t}-\operatorname{Lc}\left(f_{t}, J, \prec\right) H_{\alpha^{(t)}}$ with $\operatorname{Lm}\left(f_{t+1}, J, \prec\right) \prec \operatorname{Lm}\left(f_{t}, J, \prec\right)$, and this contradicts the fact that the sequence stops at $t$. Thus we have $f=\sum_{0}^{t-1} c_{\alpha^{(i)}} H_{\alpha^{(i)}}$. To prove the uniqueness it suffices to show that the $H_{\alpha}$ 's are linearly independent over $\mathbb{K}[J]$. But this obviously follows from the fact that the polynomials $H_{\alpha}$ have pair-wise distinct leading monomials with respect to $J$ and $\prec$.

As a consequence of Lemma 3.1 we get the following extension of Lazard's structure theorem [13. A similar result is proven in 9, 14 for radical zero-dimensional ideals and $J=x_{1}, \ldots, x_{n-1}$.

Theorem 3.2. Let $\mathcal{I}$ be a zero-dimensional ideal of $\mathbb{K}[x], J$ be a sub-list of $x$ and $\prec$ be a block order with respect to $J$. Assume that $\mathcal{I} \cap \mathbb{K}[J]$ is radical and let $G=\left\{B_{j, i} ; i=0, \ldots, s, j=1, \ldots, r_{i}\right\}$ be a Gröbner basis of $\mathcal{I}$ with respect to $\prec$, with $\operatorname{Lm}\left(B_{j, i}, J, \prec\right)=x^{\alpha^{(i)}}$. Then one can construct polynomials $H_{0}, \ldots, H_{s}$ such that:

i) $\operatorname{Lm}\left(H_{i}, J, \prec\right)=x^{\alpha^{(i)}}$ and $\operatorname{Lc}\left(H_{i}, J, \prec\right)=1$,

ii) for any $c \in \mathcal{J}\left(\mathcal{I}, J, \prec, x^{\alpha^{(i)}}\right)$ we have $c H_{i} \in \mathcal{I}$,

iii) the set $G_{1}=\left\{\operatorname{Lc}\left(B_{j, i}, J, \prec\right) H_{i} ; i=0, \ldots, s, j=1, \ldots, r_{i}\right\}$ is a Gröbner basis of the ideal $\mathcal{I}$ with respect to $\prec$.

Proof. For any $i=0,1, \ldots, s$ let $H_{i}$ be a polynomial satisfying the properties of lemma 3.1, with $x^{\alpha}=x^{\alpha^{(i)}}$. On the other hand, following the construction of $G_{1}$ we have

$$
\begin{aligned}
& \left\{\operatorname{Lc}(f, J, \prec) ; f \in G_{1}, \operatorname{Lm}(f, J, \prec) \mid x^{\alpha}\right\} \\
& =\left\{\operatorname{Lc}\left(B_{j, i}, J, \prec\right) ; x^{\alpha^{(i)}} \mid x^{\alpha}, j=1, \ldots, r_{i}\right\}
\end{aligned}
$$

for any monomial $x^{\alpha}$, and therefore this system is a Gröbner basis of $\mathcal{J}\left(\mathcal{I}, J, \prec, x^{\alpha}\right)$ according to Theorem 2.2 and to the fact that $G$ is a Gröbner basis of $\mathcal{I}$ with respect to $\prec$. By applying once again Theorem 2.2 we deduce that $G_{1}$ is a Gröbner basis of $\mathcal{I}$ with respect to $\prec$.

Another consequence of Lemma 3.1 is the following extension of Becker's result [4].

Theorem 3.3. Let $\mathcal{I}$ be a zero-dimensional ideal of $\mathbb{K}[x], J$ be a sub-list of $x$ and $\prec$ be a block order with respect to $J$. Assume that $\mathcal{I} \cap \mathbb{K}[J]$ is radical. Let $G$ be a Gröbner basis of $\mathcal{I}$ with respect to $\prec$ and let $\phi: \mathbb{K}[J] \longrightarrow \mathbb{F}$ be a ring homomorphism, where $\mathbb{F}$ is a field extension of $\mathbb{K}$. Then $\phi(G)$ is a Gröbner basis, with respect to $\prec_{J^{c}}$, of the ideal generated by $\phi(\mathcal{I})$ in $\mathbb{F}\left[J^{c}\right]$. 
Proof. Let $G$ be a Gröbner basis of $\mathcal{I}$ with respect to $\prec$. Let $p \in \mathcal{I}, x^{\alpha}=\operatorname{Lm}(p, J, \prec)$ and $c=\operatorname{Lc}(p, J, \prec)$. By applying reduction with respect to $G$ to $p$ until the monomials which are multiples of $x^{\alpha}$ are all cancelled, we get a relation of the form

$$
p=u_{1} B_{1}+\cdots+u_{t} B_{t}+R,
$$

where $B_{i} \in G, \operatorname{Lm}\left(B_{i}, J, \prec\right) \cdot \operatorname{Lm}\left(u_{i}, J, \prec\right)=x^{\alpha}$ and $\operatorname{Lm}(R, J, \prec) \prec x^{\alpha}$. In particular, if $\phi(c) \neq 0$, then there exists $i$ such that $\operatorname{Lm}\left(\phi\left(B_{i}\right), \prec\right)=x^{\alpha}$, i.e.

$$
\phi\left(\operatorname{Lc}\left(B_{i}, J, \prec J^{c}\right)\right) \neq 0 .
$$

Now let $\left(H_{\alpha}\right)_{x^{\alpha} \in \mathbb{M}\left[J^{c}\right]}$ be a sequence of polynomials satisfying the properties of lemma 3.1. Let $p \in \mathcal{I}$ and write $p=\sum_{\alpha} c_{\alpha} H_{\alpha}$, with $c_{\alpha} \in \mathcal{J}\left(\mathcal{I}, J, \prec, x^{\alpha}\right)$. Since the $H_{\alpha}$ have pairwise distinct leading coefficients we have $\operatorname{Lm}\left(\phi(p), \prec_{J^{c}}\right)=x^{\beta}$, where $x^{\beta}$ is the highest monomial with $\phi\left(c_{\beta}\right) \neq 0$.

The fact that $c_{\beta} H_{\beta} \in \mathcal{I}$ and $\phi\left(c_{\beta}\right) \neq 0$ implies the existence of $B \in G$ such that $\phi(\operatorname{Lc}(B, J, \prec)) \neq 0$ and $\operatorname{Lm}(B, J, \prec) \mid x^{\beta}$. This proves that $\operatorname{Lm}\left(\phi(B), \prec J^{c}\right) \mid$ $\operatorname{Lm}\left(\phi(p), \prec_{J^{c}}\right)$, and hence $\phi(p)$ is reducible by $\phi(G)$. This is enough to show that $\phi(G)$ is a Gröbner basis of the ideal $\phi(\mathcal{I})$ with respect to $\prec_{J^{c}}$.

\section{Counterexamples to the general case}

In this section we give examples of zero-dimensional nonradical ideals for which the results of section 3 do not hold. Symbolic computations of this section are performed using Singular [11] and Maple 9.

Consider the ideal $\mathcal{I}$ generated by the following list of polynomials:

$$
\begin{gathered}
p_{1}=t^{3}+z y-z^{2}, \quad p_{2}=x t^{3}-y^{3}, \\
p_{3}=y t^{2}-x^{2}-y^{2}, \quad p_{4}=z t+y+z^{2} .
\end{gathered}
$$

The reduced Gröbner basis $G$ of $\mathcal{I}$ with respect to the lexicographic order $x \prec y \prec$ $z \prec t$ contains 16 polynomials.

After substituting 0 to $x$ in the $p_{i}$ 's we obtain an ideal $\mathcal{I}_{0}$ whose reduced Gröbner basis is

$$
G_{0}=y^{3}, z y^{2}, y z^{2}, z^{4}+z^{3}+2 y^{2}, y^{2} t, z t+y+z^{2}, y t^{2}-y^{2}, t^{3}+z y-z^{2} .
$$

On the other hand, if we substitute 0 to $x$ in the elements of $G$, remove the contents and reduce, we get the following system:

$$
\begin{aligned}
& G_{1}=y^{3}, y^{2} z^{2}, z^{3} y, z^{4}+z^{3}+y z^{2}+2 y^{2},-y z^{2}+z y^{2}, y^{2} t, z t+z^{2}+y, \\
& y t^{2}-y^{2}, t^{3}-z^{2}+z y .
\end{aligned}
$$

Clearly, the polynomial $z y^{2} \in G_{0}$ cannot be reduced to 0 by using $G_{1}$, which proves that $G_{1}$ is not a Gröbner basis of $\mathcal{I}_{0}$.

The above example shows that Theorem 3.3 does not hold in the general case. Also, the decomposition of Lemma 3.1 does not hold since Theorem 3.3 is one of its consequences.

Next we give an example for which Theorem 3.2 does not hold but Theorem 3.3 holds. Consider the following system of polynomials:

$$
f=z^{3}-x^{2}, g=z^{3}+x y^{2}+x^{2}, h=z^{3}+x y z-y^{3} .
$$


A Gröbner basis $G$ of $\mathcal{I}=\mathcal{I}(f, g, h)$ with respect to the lexicographic order $x \prec y \prec z$ consists of the following elements:

$$
\begin{aligned}
& B_{1}=8 x^{7}-47 x^{6}+152 x^{5}+192 x^{4}+512 x^{3}, \\
& B_{2}=8192 y x^{2}+168 x^{6}-1051 x^{5}+2032 x^{4}+7744 x^{3}, \\
& B_{3}=y^{2} x+2 x^{2}, \\
& B_{4}=2048 y^{5}+168 x^{6}-1051 x^{5}+2032 x^{4}+7744 x^{3}, \\
& B_{5}=16384 z x^{2}+8192 y^{4}+168 x^{6}-1051 x^{5}+2032 x^{4}+7744 x^{3}, \\
& B_{6}=z y x-y^{3}+x^{2}, \\
& B_{7}=8192 z^{2} y^{3}+4096 z y^{4}-232 x^{6}-109 x^{5}+1680 x^{4}-14400 x^{3}, \\
& B_{8}=z^{3}-x^{2} .
\end{aligned}
$$

If we let $J=x$, then we have $\mathcal{J}(\mathcal{I}, J, \prec, z)=\mathcal{I}\left(x^{2}\right)$. Now if there exists a polynomial $H_{z}=z+q(x, y)$ such that $x^{2} H_{z} \in \mathcal{I}$, then we have $p=16384 x^{2} H_{z}-$ $B_{5} \in \mathcal{I} \cap \mathbb{K}[x, y]$, and so $p=\sum_{1}^{4} u_{i} B_{i}$. By substituting 0 to $x$ in this equality we get $y^{4}=a(y) y^{5}$, which is impossible. On the other hand, a direct computation shows that Theorem 3.3 holds for this example.

\section{REFERENCES}

1. W. W. Adams, A. Boyle, and P. Loustaunau, Transitivity for weak and strong Gröbner bases, J. Symbolic Comput. 15 (1993), no. 1, 49-65. MR1210447 (94b:68064)

2. W. W. Adams and P. Loustaunau, An introduction to Gröbner Bases, Graduate Texts in Mathematics, vol. 3, AMS, 1994. MR,1287608 (95g:13025)

3. _ Gröbner bases and primary decomposition in polynomial rings in one variable over Dedekind domains, J. Pure and Applied Algebra 121 (1997), 1-15. MR.1471120(98i:13049)

4. T. Becker, On Gröbner bases under specialization, Appl. Algebra Engrg. Comm. Comput. 5 (1994), no. 1, 1-8. MR1250930 (94h:13019)

5. T. Becker and V. Weispfenning, Gröbner Bases: A computational approach to commutative algebra, Springer-Verlag, Berlin and New York, 1993. MR.1213453 (95e:13018)

6. B. Buchberger, Ein algorithmus zum auffinden der basiselemente des restklassenringes nach einem nulldimensionalen polynomideal, Ph.D. thesis, Inst. University of Insbruck, Innsbruck, Austria, 1965.

7. _ Gröbner bases: An algorithmic method in polynomial ideal theory, In Recent trends in multidimensional system theory. Bose Ed. Reidel (1985), 184-232.

8. D. Cox, J. Little, and D. O'Shea, Ideals, varieties, and algorithms, second ed., Springer-Verlag, 1997. MR1417938 (97h:13024)

9. S. Gao, V. M. Rodrigues, and J. Stroomer, Gröbner basis structure of finite sets of points, Preprint, Available at http://www.math.clemson.edu/ sgao/pub.html (2003).

10. P. Gianni, Properties of Gröbner bases under specialization, Lect. N. Comp. Sci. Berlin, Heidelberg, New York: Springer 378 (1987), 293-297. MR.1033305 (91g:13032)

11. G.-M. Greuel, G. Pfister, and H. Schönemann, Singular 3.0, A Computer Algebra System for Polynomial Computations, Centre for Computer Algebra, University of Kaiserslautern, June, 2005, http://www.singular.uni-kl.de

12. M. Kalkbrenner, Solving systems of algebraic equations using Gröbner bases, Lect. N. Comp. Sci. Berlin, Heidelberg, New York: Springer 378 (1987), 282-292.

13. D. Lazard, Ideal bases and primary decomposition: Case of two variables, J. Symbolic Computation 1 (1985), 261-270. MR849035 (87k:13001)

14. M. G. Marinari and T. Mora, A remark on a remark by Macaulay or enhancing Lazard structural theorem, Bull. Iranian Math. Soc. 29 (2003), no. 1, 1-45, 85. MR2046304(2004m:13071)

15. B. Sturmfels, Solving systems of polynomial equations, CBMS Regional Conference Series in Mathematics, vol. 97, Published for the Conference Board of the Mathematical Sciences, Washington, DC, 2002. MR 1925796 (2003i:13037) 
Max-Planck-Institut für Informatik, Stuhlsatzenhausweg 85, 66123 Saarbrücken, Germany

E-mail address: elkahoui@mpi-sb.mpg.de

Current address: Department of Mathematics, Faculty of Sciences Semlalie, Cadi Ayyad University, P.O. Box 2390, Marrakech, Morocco

Department of Mathematics and Computer Science, Faculty of Sciences and Techniques, Cadi Ayyad University, P.O. Box 549 Marrakech, Morocco

E-mail address: rakrak@fstg-marrakech.ac.ma 